\title{
Effectiveness and Tolerability of Fondaparinux vs Enoxaparin in a Population of Indian Patients with Symptomatic Deep Vein Thrombosis: A Retrospective Real-World Study
}

\author{
Pinjala Ramakrishna ${ }^{1} \cdot$ P. C. Gupta ${ }^{2} \cdot$ Paresh Pai $^{3} \cdot$ Kumud Rai $^{4} \cdot$ M. Rajkumar ${ }^{5} \cdot$ Tapish Sahu $^{6}$. \\ Mohammed Yunus Khan ${ }^{7} \cdot$ Kumar Gaurav $^{7}$. Amey Mane ${ }^{7}$. Sucheta Pandit ${ }^{7} \cdot$ Madhur Jain $^{8} \cdot$ Anand Subramaniyan $^{9}$
}

Accepted: 28 July 2021 / Published online: 25 August 2021

(c) The Author(s) 2021

\begin{abstract}
Background Fondaparinux is the first approved anticoagulant drug among factor Xa inhibitors, with proven effectiveness and safety in preventing deep vein thrombosis. However, limited data are available supporting the benefit-risk profile of fondaparinux vs enoxaparin in a real-world group of Indian patients with deep vein thrombosis.

Objective To compare the effectiveness and tolerability of fondaparinux vs enoxaparin in patients with symptomatic deep vein thrombosis in a long-term real-world setting.

Methods Data from the electronic medical records of adult patients diagnosed with deep vein thrombosis prescribed fondaparinux $(n=503)$ or enoxaparin $(n=508)$ as monotherapy were analyzed. Effectiveness was analyzed in terms of recurrence, duration, and type of deep vein thrombosis event, and tolerability as bleeding events at initial hospitalization and follow-up visits up to 3 months duration. Appropriate statistical methods were used to determine the significance $(p<0.05)$ between the two groups.

Results The deep vein thrombosis recurrence in the fondaparinux group was non-inferior (2.78\%) when compared with enoxaparin (3.76\%), with a mean duration of 47 and 48 days, respectively. The number of events and mean duration of events (in days) were not significant $(p>0.05)$. Major bleeding events were higher in the enoxaparin group at $3.17 \%$ than the fondaparinux group at $2.19 \%$, and the difference was not statistically significant $(p>0.05)$.

Conclusions The weight-based, once-daily subcutaneous fondaparinux dose showed non-inferior effectiveness and a comparable tolerability profile when compared with the twice-daily enoxaparin dose for the management of symptomatic deep vein thrombosis.
\end{abstract}

\section{Introduction}

Deep vein thrombosis (DVT) and pulmonary embolism (PE) are the two most crucial subsets of venous thromboembolism (VTE) and are the primary preventable cause of morbidity and mortality worldwide [1-3]. Venous thromboembolism can occur as just DVT only, or as PE with DVT, or PE without DVT [2]. Venous thromboembolism is the third most usual life-threatening cardiovascular disease after stroke and myocardial infarction [3, 4]. Indications

Mohammed Yunus Khan

doctorkhan26@gmail.com

Extended author information available on the last page of the article

\section{Key Points}

Therapy with a pre-filled injection dose of fondaparinux had a non-inferior effectiveness and comparable tolerability to the twice-daily enoxaparin dose in terms of reductions in thrombosis and major bleeding events.

This study adds to the existing information regarding fondaparinux with respect to low-molecular-weight heparin (enoxaparin) in terms of efficacy and tolerability in real-world practice. 
and clinical signs reminiscent of DVT are normal in the general population and have various potential causes [1].

Deep vein thrombosis is a common condition affecting about 1-2 per 1000 adults annually that arises quickly following age 45 years [2]. Deep vein thrombosis leads to the formation of PE when a blood clot (or multiple clots) that forms inside deep veins in the lower limb disconnect from the veins (embolize) to the vena cava, right atrium, and right ventricle and lodge in pulmonary arteries that supply deoxygenated blood to the lungs for oxygenation [2]. This blockage of the bloodstream to the lungs is called $\mathrm{PE}$, occurs in up to $33 \%$ of cases, and is the essential contributor to mortality. The clinical conditions most usually associated with DVT are medical procedures or injury, prolonged immobility, malignancy, congestive heart failure, pregnancy, varicose vein, advancing age, history of DVT, and obesity [5].

Anticoagulants are the mainstay of DVT treatment, aimed at preventing progression to PE and recurrence of thrombosis [5]. Traditionally, the anticoagulation approach for the treatment of acute VTE has for most patients involved a parenteral anticoagulant, for example, low-molecular-weight heparin (LMWH) or fondaparinux, overlapping with and followed by a vitamin $\mathrm{K}$ antagonist or novel oral anticoagulants for long-term treatment [4]. Enoxaparin is one of the anticoagulants that belongs to the LMWH family of medications. It irreversibly inactivates clotting factor $\mathrm{Xa}$ and helps prevent the formation of blood clots. In contrast to prophylaxis with unfractionated heparin, LMWH has shown effectiveness in reducing the rates of DVT/PE by about $60 \%$ [6, 7]. In comparison with unfractionated heparin, LMWH appears more effective in preventing VTE among patients with stroke [8].

While for several years the standard therapy of LMWH has been in use, the trend has been changing towards factor Xa inhibitors (fondaparinux) because of their selectivity in action [9]. Fondaparinux was the first approved drug among factor Xa inhibitors [10] and is a synthetic pentasaccharide that selectively binds to antithrombin, making it rapidly inhibit factor Xa, a key enzyme in the coagulation pathway [9-18]. Pharmacokinetic analysis suggested that fondaparinux has rapid and complete absorption and a longer half-life, resulting in 24-h coverage without crossreacting with heparin-induced antibodies, and no need for monitoring of platelet counts [9-15].

The noninferiority of fondaparinux over enoxaparin could be correlated with its ability to initiate selective inhibition of factor Xa, its predictable linear pharmacokinetics with $100 \%$ bioavailability (subcutaneous route), its pharmacological characteristics (in which it is not considered a heparin-related molecule and does not appear to cause heparininduced thrombocytopenia with no effect on platelet function and aggregation), the minimal risk of drug interactions, and its long duration of action for 17-21 h, which make it a drug of choice for symptomatic DVT.

Several trials have shown the benefit-risk ratio of fondaparinux over enoxaparin in preventing VTE in various patient profiles. Much of the evidence was either generated from randomized or specific cohort studies, under controlled clinical conditions [4, 6, 12-15, 18]. Hence, it is important to re-evaluate the performance of fondaparinux under uncontrolled clinical conditions such as real-world settings. Nevertheless, there were some limited data showing the same results could be reproduced in a general population in regular practice with no specific selection criteria. Additionally, the net clinical benefit-risk description of this anticoagulant, enoxaparin as a comparator, has not been comprehensively studied in a real-world group of Indian patients with DVT or, even if studied, has insufficient evidence available. Therefore, in the present study, we compared the effectiveness and tolerability of a once-daily subcutaneous dose regimen of fondaparinux with that of a twice-daily subcutaneous dose regimen of enoxaparin in long-term real-world clinical practice in an Indian population.

\section{Methods}

In this retrospective, longitudinal, real-world, observational study, data were collected from the department of cardiology of multiple tertiary care centers, over a period of 5 years, starting from June 2015 to May 2020. A total of 1018 electronic medical records (EMRs) were screened for the selection of the study population. Among those patients who had met all the inclusion criteria, there were 503 in the fondaparinux group and 505 in the enoxaparin group (Fig. 1).

An independent ethics committee located in Pune, India approved the study protocol. This was a retrospective study that used the anonymized or anonymous data (existing medical records available as of the date of the independent ethics committee submission) without any additional prospective components for research purposes. Hence, the process did not necessitate obtaining informed consent because the study did not involve identifiable individuals. Accordingly, the independent ethics committee gave permission for the informed consent form waiver before the initiation of the data collection process for this study. Adult patients ( $>18$ years of age) who presented with an established diagnosis of symptomatic DVT (involving the popliteal, femoral, or iliac veins or the trifurcation of the calf veins or any other distal vein) confirmed by venous ultrasound and/or computed tomography scan and who required antithrombotic therapy were included in the study.

Patients were excluded from the study if they had a symptomatic PE in the first visit; received a therapeutic dose of anticoagulant or oral anticoagulant therapy for more than 24 hours; required thrombolysis, thrombectomy, or vena 




Fig. 1 Sample selection flow chart for fondaparinux and enoxaparin. DVT deep vein thrombosis, $e G F R$ estimated glomerular filtration rate

cava filter; had a contraindication to anticoagulant therapy (e.g., active bleeding, thrombocytopenia [platelet count $<$ $50,000]$ ); estimated glomerular filtration rate $<30 \mathrm{~mL} / \mathrm{min}$; had a contraindication to contrast medium; had uncontrolled hypertension (systolic blood pressure $>180 \mathrm{mmHg}$ or diastolic blood pressure $>110 \mathrm{mmHg}$ ); pregnant and lactating women, or had a life expectancy of fewer than 3 months.

The medical records of the patient visit from the baseline up to a period of 90 days ( 3 months) were taken into consideration. The patients in the fondaparinux group had received a weight-based once-daily subcutaneous injection of $5 \mathrm{mg}$ if they had a body weight $<50 \mathrm{~kg}, 7.5 \mathrm{mg}$ if they had a body weight between 50 and $100 \mathrm{~kg}$, or $10 \mathrm{mg}$ if they had a body weight $>100 \mathrm{~kg}$. The patients in the enoxaparin group had received a twice-daily subcutaneous injection of $1 \mathrm{mg} / \mathrm{kg}$ of body weight and both drugs had to be given for a minimum of 5 days during hospitalization as the per-protocol requirement.

\subsection{Outcome Assessment}

The effectiveness outcomes were assessed from the incidences of recurrent symptomatic thrombosis events (DVT or $\mathrm{PE}$ ), duration of the event from the baseline (in days), and the type of recurrence (fatal PE, non-fatal PE, and DVT) at initial hospitalization and follow-up visits at 30 (first visit),
60 (second visit), and 90 (third visit) days in each group during the 3-month study period. The tolerability outcomes were evaluated by major and minor bleeding events at initial hospitalization and at the end of every follow-up visit in each group during the 3-month study period. The definitions of the outcomes are presented in the Electronic Supplementary Material.

\subsection{Statistical Analysis}

All outcomes were presented using descriptive statistics. Continuous data were expressed as mean and standard deviation and categorical data as numbers and percentages. The comparison of mean differences of the data was analyzed by a $T$-test and categorical variables by a Chi-square test. A $p$-value of $<0.05$ was considered statistically significant.

\section{Results}

\subsection{Baseline Demographic Characteristics}

The demographic parameters were comparable between the two groups (Table 1). The overall mean age for the fondaparinux group was $57.21( \pm 8.86)$ years, and $57.78( \pm$ 9.57) years for the enoxaparin group. The proportions of 
male and female individuals were comparable between both groups, fondaparinux [male $70.97 \%(n=357)$ and female $29.02 \%(n=146)$ ] and enoxaparin [male $70.69 \%(n=357)$ and female $29.30 \%(n=148)]$. The mean bodyweight was comparable between the two groups under the $50-100 \mathrm{~kg}$ category $(87.67 \%$ of patients in the fondaparinux group vs
Table 1 Baseline demographic characteristics $(n=1008)$

\begin{tabular}{|c|c|c|c|}
\hline Parameters & $\begin{array}{l}\text { Fondaparinux } \\
n=503\end{array}$ & $\begin{array}{l}\text { Enoxaparin } \\
n=505\end{array}$ & $p$ Value \\
\hline \multicolumn{4}{|l|}{ Demographic profile } \\
\hline Age (in years), mean (SD) & $57.21 \pm 8.86$ & $57.78 \pm 9.57$ & 0.32 \\
\hline \multicolumn{4}{|l|}{ Sex, $n(\%)$} \\
\hline Male & 357 (70.97) & $357(70.69)$ & 0.92 \\
\hline Female & $146(29.02)$ & $148(29.30)$ & - \\
\hline \multicolumn{4}{|l|}{ Weight (kg), mean (SD) } \\
\hline$<50$ & $16(3.18)$ & $13(2.57)$ & 0.564 \\
\hline $50-100$ & $441(87.67)$ & $442(87.52)$ & 0.943 \\
\hline$>100$ & $46(9.15)$ & $50(9.90)$ & 0.682 \\
\hline Height $(\mathrm{cm})$, mean $(\mathrm{SD})$ & $161.89 \pm 4.17$ & $161.66 \pm 3.88$ & 0.364 \\
\hline Pulse (BPM), mean (SD) & $82.91 \pm 12.13$ & $79.65 \pm 11.81$ & 0.0001 \\
\hline $\mathrm{SBP}(\mathrm{mmHg})$, mean $(\mathrm{SD})$ & $118.62 \pm 18.33$ & $116 \pm 17.87$ & 0.022 \\
\hline $\mathrm{DBP}(\mathrm{mmHg})$, mean $(\mathrm{SD})$ & $75.41 \pm 12.33$ & $74.14 \pm 12.34$ & 0.102 \\
\hline \multicolumn{4}{|l|}{ Comorbidities, $n$ (\%) } \\
\hline Diabetes mellitus & $97(19.28)$ & $90(17.82)$ & 0.550 \\
\hline Heart failure & $86(17.09)$ & $87(17.22)$ & 0.956 \\
\hline ICU stay and immobilization & $83(16.50)$ & $78(15.44)$ & 0.647 \\
\hline CAD & $78(15.51)$ & $78(15.44)$ & 0.974 \\
\hline Dyslipidemia & $63(12.52)$ & $60(11.88)$ & 0.755 \\
\hline Obesity & $59(11.72)$ & $61(12.08)$ & 0.864 \\
\hline Active cancer & $22(4.37)$ & $21(4.16)$ & 0.864 \\
\hline Surgery/trauma ( $<3$ months) & $22(4.37)$ & $23(4.55)$ & 0.890 \\
\hline Chemotherapy/radiotherapy & $22(4.37)$ & $21(4.15)$ & 0.864 \\
\hline Varicose vein & $14(2.78)$ & $18(3.56)$ & 0.479 \\
\hline Stroke & $9(1.78)$ & $6(1.18)$ & 0.431 \\
\hline Chronic kidney disease & $4(0.79)$ & $6(1.188)$ & 0.755 \\
\hline Other $^{\mathrm{a}}$ & $1(0.19)$ & $2(0.39)$ & 0.565 \\
\hline \multicolumn{4}{|l|}{ Lifestyle related } \\
\hline Smokers & $82(16.30)$ & $72(14.25)$ & 0.367 \\
\hline Alcoholics & $78(15.50)$ & $76(15.05)$ & 0.839 \\
\hline \multicolumn{4}{|l|}{ Medical history } \\
\hline Past history of DVT & $41(8.15)$ & $42(8.32)$ & 0.924 \\
\hline Family history of DVT & $83(16.50)$ & $86(17.03)$ & 0.823 \\
\hline Mother & $43(8.55)$ & $43(8.55)$ & - \\
\hline Father & $19(3.78)$ & $18(3.58)$ & - \\
\hline Other (brother and sister) & $20(3.98)$ & $25(4.97)$ & - \\
\hline Other family history & $88(17.50)$ & $93(18.42)$ & 0.703 \\
\hline Hypertension & $51(10.14)$ & $56(11.13)$ & - \\
\hline Hypothyroidism & $32(6.36)$ & $32(6.36)$ & - \\
\hline CAD & $2(0.40)$ & $1(0.20)$ & - \\
\hline No details (no data) & $3(0.60)$ & $4(0.80)$ & - \\
\hline $\begin{array}{l}\text { Time between onset of symptoms and initia- } \\
\text { tion of DVT treatment (in days) }\end{array}$ & $1.59 \pm 0.80$ & $1.56 \pm 0.84$ & 0.0001 \\
\hline
\end{tabular}

$B P M$ beats per minute, $C A D$ coronary artery disease, $D B P$ diastolic blood pressure, $D V T$ deep vein thrombosis, $I C U$ intensive care unit, $S B P$ systolic blood pressure, $S D$ standard deviation

${ }^{a}$ Known prothrombotic state, hyperhomocysteinemia, antiphospholipid antibodies 
$87.52 \%$ patients in the enoxaparin group), whereas in the $<$ $50 \mathrm{~kg}$ category, it was $3.18 \%$ of patients in the fondaparinux group and $2.57 \%$ of patients in the enoxaparin group, and in the $>100 \mathrm{~kg}$ category, it was $9.15 \%$ of patients in the fondaparinux group and $9.90 \%$ of patients in the enoxaparin group. The once-daily fondaparinux injection and the twicedaily enoxaparin injection mean duration in days was 5.27 \pm 0.97 (standard deviation). The minimum duration of both the drugs was 3 days and the maximum duration was 9 days.

\subsection{DVT Type and Location Details at Baseline}

All patients were reported to have unilateral thrombosis (Table 2). Thrombosis was reported in proximal veins, the femoral and trifurcation veins in about $20 \%$ of the patients in both the groups. Iliac was of $19 \%$ each and popliteal was $16 \%$ and $18.6 \%$ in the fondaparinux and enoxaparin groups, respectively. While in distal veins, peroneal veins were majorly reported thrombosis with $47 \%$ and $45 \%$ in fondaparinux and enoxaparin, respectively.

\subsection{Effectiveness Outcomes}

The recurrent incidences of DVT in patients treated with fondaparinux was lower than in patients treated with enoxaparin (Fig. 2A). The total episodes of clinically suspected recurrence of symptomatic VTE in the fondaparinux and enoxaparin groups were 14 (2.78\%) and 19 (3.76\%), respectively, among which major episodes were reported in 3 months [13 (2.58\%) in the fondaparinux group and $17(3.37 \%)$ in the enoxaparin group] from the baseline and minimal episodes $(n=1)$ during the hospitalization (fondaparinux group) and the first and second months (enoxaparin group). The difference in events between the groups was not statistically significant $(p>0.05)$.

The duration of a recurrent event (in days) was after 30 (one event), 61 (one event), and 88 (17 events) days in the

Table 2 Deep vein thrombosis (DVT) type and location details $(n=$ 1008)

\begin{tabular}{lccc}
\hline DVT details & $\begin{array}{c}\text { Fondaparinux } \\
n=503, n(\%)\end{array}$ & $\begin{array}{l}\text { Enoxaparin } \\
n=505, n(\%)\end{array}$ & $p$ Value \\
\hline Iliac & $94(18.68)$ & $99(19.60)$ & 0.711 \\
Femoral & $103(20.47)$ & $108(21.38)$ & 0.722 \\
Popliteal & $83(16.50)$ & $94(18.61)$ & 0.378 \\
Trifurcation & $102(20.27)$ & $100(19.80)$ & 0.849 \\
Anterior tibial & $74(14.71)$ & $69(13.66)$ & 0.633 \\
Posterior tibial & $139(27.63)$ & $140(27.73)$ & 0.974 \\
Peroneal & $237(47.11)$ & $231(45.73)$ & 0.662 \\
Muscular vein & $33(6.56)$ & $36(7.13)$ & 0.720 \\
Bilateral & $2(0.39)$ & $2(0.39)$ & 0.619 \\
Unilateral & $501(99.60)$ & $503(99.60)$ & - \\
\hline
\end{tabular}

enoxaparin group and 5 (one event) and 89 (13 events) days for the fondaparinux group (Fig. 2B). The difference in the total duration of event occurrence between two groups was statistically not significant $(p>0.05)$. Only DVT events were recurrently reported in the study sample for both the drugs. No fatal and non-fatal PE events were reported (Fig. 3).

\subsection{Tolerability Outcome}

The major bleeding events were numerically higher in the enoxaparin group, $16(3.17 \%)$ [11 $(2.19 \%)$ in the fondaparinux group], among which two events $(0.40 \%)$ occurred during the hospitalization and ten events $(1.98 \%)$ occurred during the first follow-up visit (at 30 days), followed by four $(0.79 \%)$ events at the time of the second visit (at 60 days) (Fig. 4). The minor bleeding events were numerically higher in the fondaparinux group, $47(9.3 \%)$ [43 (8.51\%) in the enoxaparin group], among which 19 events (3.78\%) occurred during the initial hospitalization treatment and 20 events (3.98\%) occurred during the first follow-up visit (at 30 days), followed by eight (1.59\%) events during the second visit (at 60 days). None of the events between the groups was statistically significant $(p>0.05)$.

\subsection{Impact of Bodyweight}

The majority of the thromboembolism (Fig. 5) and major bleeding events (Fig. 6) occurred in the ' $50-100$ kg' body weight category of the study population. The incidences of major events were reported during the follow-up period from the baseline and the minimal events $(n=1)$ were reported during the hospitalization.

\section{Discussion}

The occurrence of DVT is followed by several thrombotic factors that promote the formation of a new clot and/or event that would, in turn, re-establish the venous lumen [29]. Thus, DVT is rather difficult to prevent with only anticoagulant therapy, as one of the most important challenges in its management is to deal with its considerable recurrence risk [18-22]. Although prolonged treatment of DVT with anticoagulant therapy offers effective prevention of the recurrence, because of its associated bleeding risk it is not universally acceptable [21, 23]. Additionally, the likelihood of either a thrombotic or bleeding event is high, and both risks will persist over an individual's lifetime [24, 25]. The pentasaccharide fondaparinux is the first of the new class of synthetic antithrombotic agents that acts through specific inhibition of factor Xa, devoid of direct activity against thrombin (factor IIa). This inactivation of factor Xa via antithrombin results in active inhibition of thrombin generation [18], while the 
A

Recurrence Events (n) of DVT



Study Timelines

B Duration (in days) of Recurrent Event of DVT $p$-value $>0.05$

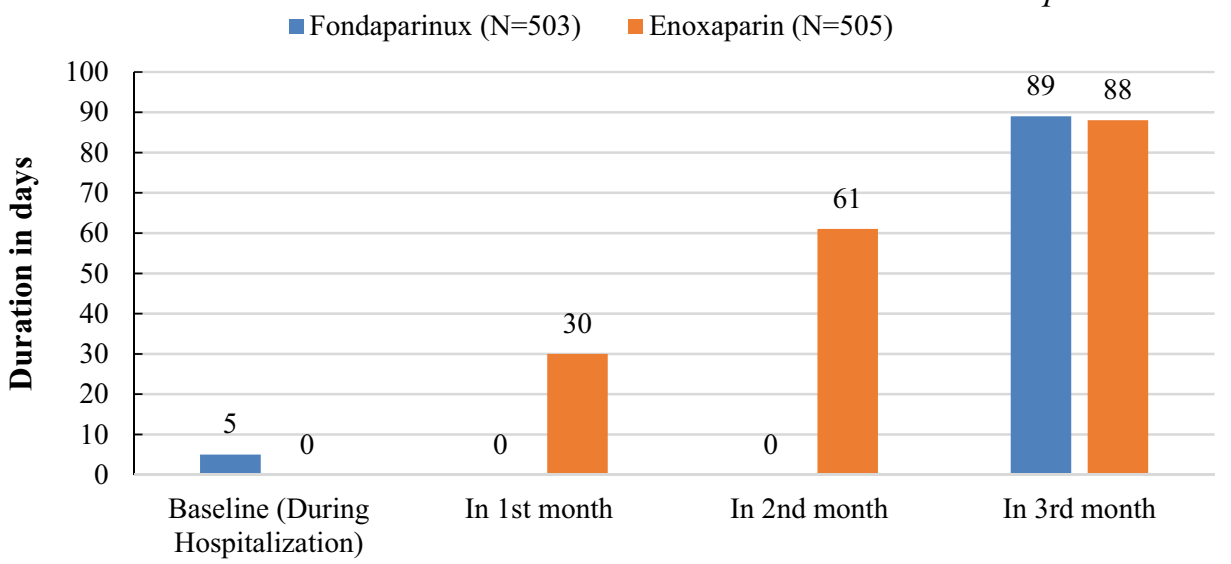

Study Timelines

Fig. 2 Number of deep vein thrombosis (DVT) recurrence events $[n(\%)]$ are given in the top panel; duration of event from baseline is given in the bottom panel

LMWH drug enoxaparin acts by binding to antithrombin and irreversibly inactivates clotting factor $\mathrm{Xa}$ [6].

The study objective was to compare the effectiveness and tolerability parameters of weight-based once-daily subcutaneous fondaparinux to those of twice-daily per body weight subcutaneous enoxaparin, for recurrent symptomatic DVT in a real-world patient. The study results demonstrated the noninferiority effectiveness of fondaparinux when compared with enoxaparin in terms of the number of events and the duration of an event from the baseline. During a followup of 90 days, the total number of recurrent events were numerically lower in fondaparinux [14] when compared with enoxaparin [19] $(p>0.05)$. The type of recurrent events assessed as fatal and non-fatal PE events was not reported in both of the treatment groups.

Similar results were observed by Bauer et al., wherein the efficacy and safety of fondaparinux were compared with enoxaparin for the initial treatment of symptomatic DVT and reported a significantly lower incidence of VTE $(12.5 \%)$ in the fondaparinux group than the enoxaparin group $(27.8 \%)$ [ $p<0.06$ ] [6]. A comparative outcome by Migita et al. [26] in the Japanese population also demonstrated a significant reduction in the incidence of DVT in the fondaparinux group compared with the enoxaparin group. A study by Eriksson et al. demonstrated the incidence of VTE was significantly lower $(p<0.001)$ in the fondaparinux group $(8.3 \%)$ than in 




Fig. 3 Type of recurrence of deep vein thrombosis across study timelines

\section{Bleeding Event Count (n)}

- Baseline (During Hospitalization) $\square$ Events in 1st month $\square$ Events in 2nd month $\square$ Events in 3rd month

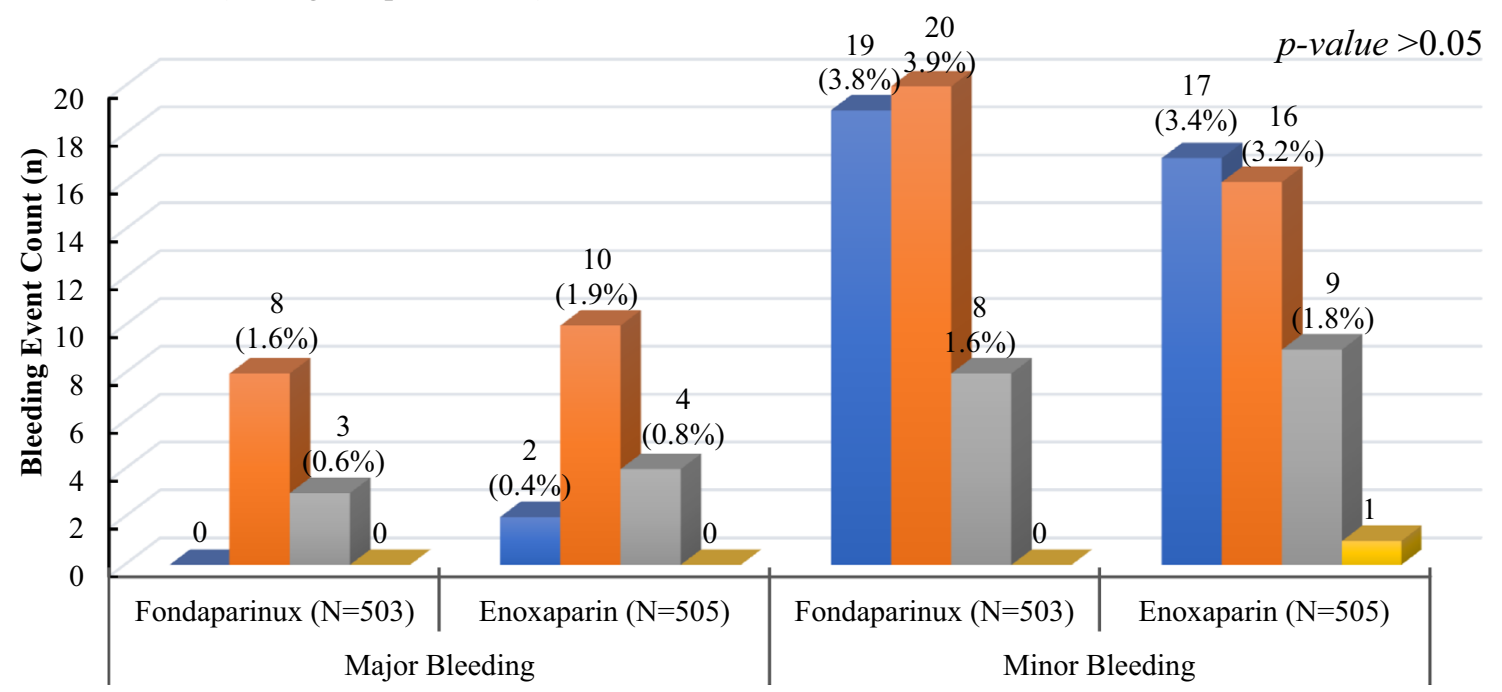

Fig. 4 Count of bleeding events is shown from baseline to the end of the study duration (third month)

the enoxaparin group (19.1\%) [27], and also reported the low incidence of symptomatic VTE and fatal and non-fatal PE with no difference between the two groups [27]. A similar investigation by Lassen et al. had also demonstrated the same conclusion exhibiting superior efficacy of fondaparinux $(p<$ 0.001 ) [10] in patients who underwent elective hip replacement surgery. A meta-analysis by Turpie et al. had likewise affirmed this observation [11]. The real-world studies also reported the same effectiveness of fondaparinux [16, 24, 26].
Major bleeding is considered the primary safety concern with the use of any anticoagulant, and the appropriate responses are important to consider $[4,6]$. In the current study, the bleeding events up to 3 months from the baseline were compared, and the fondaparinux group had a numerically lower rate of major bleeding events as compared with the enoxaparin group $(2.19 \%$ with fondaparinux and $3.17 \%$ with enoxaparin). However, the difference observed between the two treatments concerning the risk of major bleeding was 

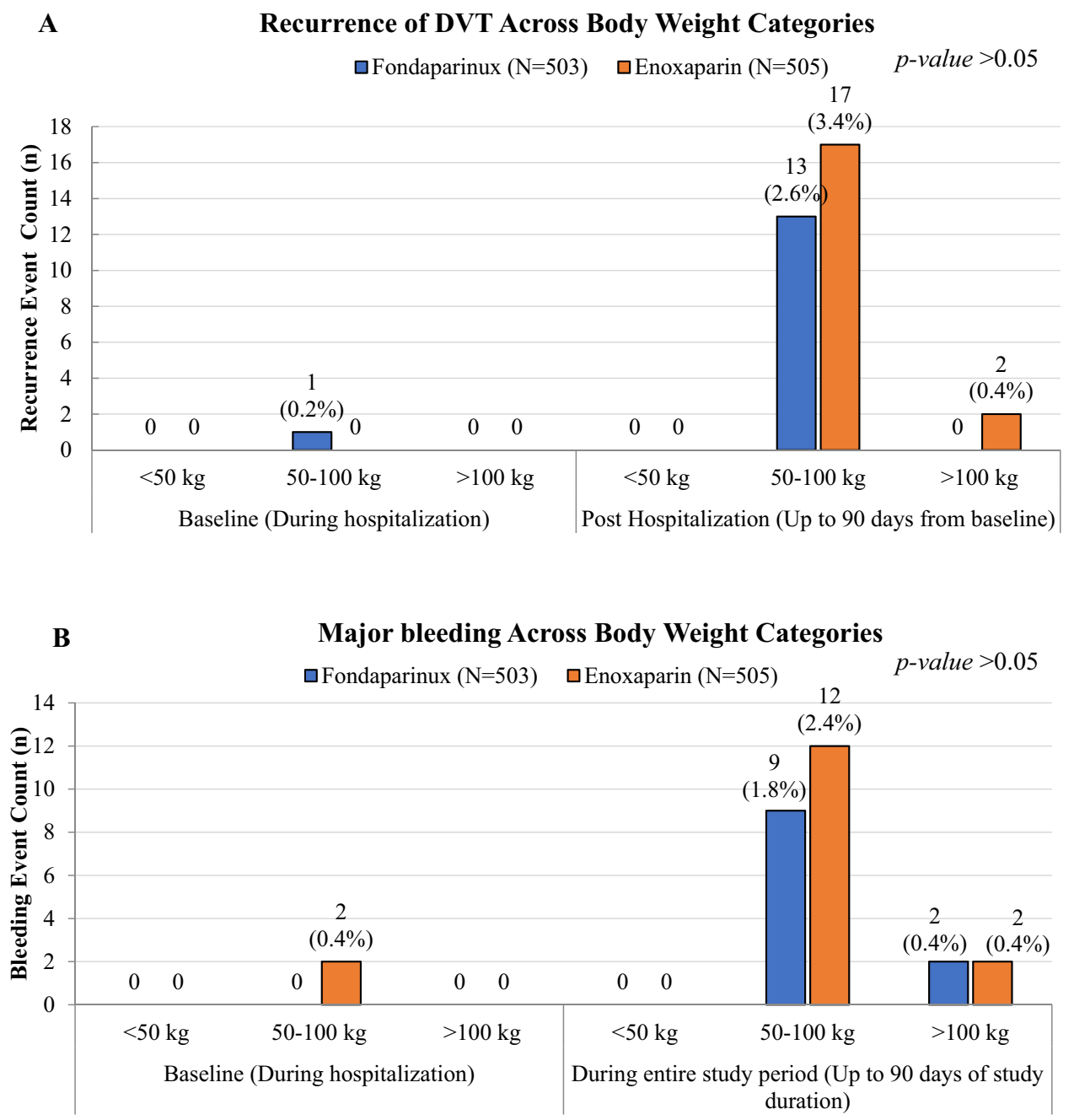

Fig. 5 Recurrence of deep vein thrombosis (DVT) events across body weight categories is given in the top panel; major bleeding events across body weight categories is given in the bottom panel

not significant $(p>0.05)$. A study by Turpie et al. reported no difference in clinically relevant bleeding between the two groups [11].

During the subgroup analyses, it seems that both the treatments had effectiveness $(2.58 \%$ in the fondaparinux group and $3.38 \%$ in the enoxaparin group) and tolerability $(1.7 \%$ in fondaparinux and $2.37 \%$ in enoxaparin) independent of the body weight. Bauer et al. reported no influence of body weight on the clinical outcomes [6], while Eichinger et al. [28] demonstrated the risk of recurrence shows an almost linear relationship with an increase in body weight. In our study, we have seen no such relationship with body weight.

The other outcomes of the present study were baseline parameters, namely age, sex, weight, and height of the study sample were comparable between the two groups; however, in the present study, the male population was predominant. In a study by Donath et al. [28], female individuals were more often in the fondaparinux group. Diabetes mellitus, coronary artery disease, heart failure, obesity, and dyslipidemia were the most common comorbidities observed. The most affected veins in DVT involved peroneal vein, posterior tibial vein, and femoral vein with no difference in both the groups. Both serum creatinine and D-dimer values were comparable in both the groups. A significant relationship between the onset of symptoms and the initiation of DVT treatment between the two drugs was observed. However, in the study by Turpie et al. [18], the relationship between the onset of symptoms and the timing of the first injection was not significant.

\subsection{Limitations}

In this retrospective study, like other retrospective EMRbased RWE studies, the EMRs contain only the prescription data, thus we cannot exclude the possibility of some patients not adhering to the treatment, leading to one or 
multiple missed doses, which could have contributed to a variation in treatment outcomes. Furthermore, there were different methods used, instead of a single protocol, to assess the recurrence of DVT at various centers, and patients were switched to oral anticoagulants after hospitalization because of the injectable form of the treatment and the cost. This might have caused a variation in the measurements. The methods used to evaluate DVT have also not been covered in the EMRs, thus no proper estimation has been made for the same.

\section{Conclusions}

In the current study, therapy with a pre-filled injection dose of fondaparinux had a non-inferior effectiveness and comparable tolerability to the twice-daily enoxaparin dose in terms of a reduction in thrombosis and major bleeding events. This EMR-based, retrospective real-world evidence study has provided additional information regarding the effectiveness and tolerability of fondaparinux towards the management of symptomatic DVT in Indian patients.

Supplementary Information The online version contains supplementary material available at https://doi.org/10.1007/s40801-021-00273-4.

Acknowledgements The authors thank Mrs. Mohini Gahlot (medical writer), Dr. Srivani Palukuri (clinical research scientist, RWE), and Dr. Saurav Deka (AVP, clinical operations) from Sekhmet Technologies Private Limited (THB), India, for their support with the preparation of the manuscript.

\section{Declarations}

Funding The study was funded by Dr. Reddy's Laboratories Ltd.

Conflicts of Interest/Competing Interests This study represents original work. It was completed recently and has not been published elsewhere. The authors, Dr. P. Ramakrishna, Dr. P.C. Gupta, Dr. P. Pai, Dr. K. Rai, Dr. Rajkumar M, and Dr. T Sahu are the advisory board members for Dr. Reddy's Laboratories Ltd. Dr. Md Y. Khan, Dr. K. Gaurav, Dr. A. Mane, and S. Pandit declare that they work in the Medical Affairs Department in Dr. Reddy's Laboratories Ltd, Hyderabad, India. Dr. M Jain and Dr. A Subramaniyan declare that there is no conflict of interest towards publishing the work.

Ethics Approval The study was approved by an independent ethics committee located in Pune, India.

Consent to Participate This was a retrospective study and used the anonymized or anonymous data (existing medical records available as of the date of the independent ethics committee submission) without any additional prospective components for research purposes. Hence, the process did not necessitate the obligation to obtain informed consent as the study did not involve identifiable individuals.

Consent for Publication Not applicable.
Availability of Data and Material All the data outcomes generated and/ or analyzed during the current study are included in this published article.

Code Availability Not applicable.

Authors' Contributions Authors from Dr. Reddy's Laboratories Ltd had contributed towards conceptualizing the study hypothesis, the design of the study, data analysis and interpretation of study outcomes, and compiling the manuscript. The advisory board members significantly contributed to critically revise the manuscript for important intellectual content and final approval and review. Dr. M. Jain and Dr. A. Subramaniyan provided the required study data inputs and the important insights on patient outcomes.

Open Access This article is licensed under a Creative Commons Attribution-NonCommercial 4.0 International License, which permits any non-commercial use, sharing, adaptation, distribution and reproduction in any medium or format, as long as you give appropriate credit to the original author(s) and the source, provide a link to the Creative Commons licence, and indicate if changes were made. The images or other third party material in this article are included in the article's Creative Commons licence, unless indicated otherwise in a credit line to the material. If material is not included in the article's Creative Commons licence and your intended use is not permitted by statutory regulation or exceeds the permitted use, you will need to obtain permission directly from the copyright holder. To view a copy of this licence, visit http://creativecommons.org/licenses/by-nc/4.0/.

\section{References}

1. Kahn SR. The clinical diagnosis of deep venous thrombosis: integrating incidence, risk factors, and symptoms and signs. Arch Intern Med. 1998;158(21):2315-23. https://doi.org/10.1001/archi nte.158.21.2315.

2. Heit JA, Spencer FA, White RH. The epidemiology of venous thromboembolism. J Thromb Thrombolysis. 2016;41(1):3-14. https://doi.org/10.1007/s11239-015-1311-6.

3. Wilbur J, Shian B. Deep venous thrombosis and pulmonary embolism: current therapy. Am Fam Physician. 2017;95(5):295-302.

4. Ageno W, Turpie AG. Spotlight on real-world evidence for the treatment of DVT. Thromb Haemost. 2016;116(Suppl. 2):S41-9. https://doi.org/10.1160/TH16-06-0488.

5. Stone J, Hangge P, Albadawi H, et al. Deep vein thrombosis: pathogenesis, diagnosis, and medical management. Cardiovasc Diagn Ther. 2017;7(Suppl. 3):S276-84. https://doi.org/10.21037/ cdt.2017.09.01.

6. Büller HR, Davidson BL, Decousus H, et al. Fondaparinux or enoxaparin for the initial treatment of symptomatic deep venous thrombosis: a randomized trial. Ann Intern Med. 2004;140(11):867-73. https://doi.org/10.7326/0003-4819-14011-200406010-00007.

7. McKenzie CR, Abendschein DR, Eisenberg PR. Sustained inhibition of whole-blood clot procoagulant activity by inhibition of thrombus-associated factor Xa. Arterioscler Thromb Vasc Biol. 1996;16:1285-91.

8. Agnelli G, Gallus A, Goldhaber SZ, et al. Treatment of proximal deep-vein thrombosis with the oral direct factor Xa inhibitor rivaroxaban (BAY 59-7939): the ODIXa-DVT (Oral Direct Factor Xa Inhibitor BAY 59-7939 in patients with acute symptomatic deepvein thrombosis) study. Circulation. 2007;116(2):180-7. https:// doi.org/10.1161/CIRCULATIONAHA.106.668020. 
9. Alexander JH, Singh KP. Inhibition of factor Xa: potential target for the development of new anticoagulants. Am J Cardiovasc Drugs. 2005;5(5):279-90. https://doi.org/10.2165/00129784$200505050-00001$.

10. Lassen MR, Bauer KA, Eriksson BI, Turpie AG, European Pentasaccharide Elective Surgery Study (EPHESUS) Steering Committee. Postoperative fondaparinux versus preoperative enoxaparin for prevention of venous thromboembolism in elective hip-replacement surgery: a randomised double-blind comparison. Lancet. 2002;359(9319):1715-20. https://doi.org/10.1016/S01406736(02)08652-X

11. Nicolaides AN, Breddin HK, Fareed J, et al. Prevention of venous thromboembolism: international consensus statement. Guidelines compiled in accordance with the scientific evidence. Int Angiol. 2001;20(1):1-37.

12. Hata K, Kimura T, Tsuzuki S, et al. Safety of fondaparinux for prevention of postoperative venous thromboembolism in urological malignancy: a prospective randomized clinical trial. Int J Urol. 2016;23:923-8.

13. Sasaki S, Miyakoshi N, Matsuura H, Saitoh H, Kudoh D, Shimada Y. Prospective randomized controlled trial on the effect of fondaparinux sodium for prevention of venous thromboembolism after hip fracture surgery. J Orthop Sci. 2009;14:491-6.

14. Agnelli G, Bergqvist D, Cohen AT, Gallus AS, Gent M. Randomized clinical trial of postoperative fondaparinux versus perioperative dalteparin for prevention of venous thromboembolism in high-risk abdominal surgery. Br J Surg. 2005;92:1212-20.

15. Steele KE, Canner J, Prokopowicz G, et al. The effort trial: preoperative enoxaparin versus postoperative fondaparinux for thromboprophylaxis in bariatric surgical patients: a randomized doubleblind pilot trial. Surg Obes Relat Dis. 2015;11:672-83.

16. Yokote R, Matsubara M, Hirasawa N, Hagio S, Ishii K, Takata C. Is routine chemical thromboprophylaxis after total hip replacement really necessary in a Japanese population? J Bone Jt Surg Br. 2011;93:251-5.

17. Bauer KA, Eriksson BI, Lassen MR, Turpie AG. Fondaparinux compared with enoxaparin for the prevention of venous thromboembolism after elective major knee surgery. N Engl J Med. 2001;345:1305-10.

18. Turpie AGG, Gallus AS, Hoek JA. A synthetic pentasaccharide for the prevention of deep vein thrombosis after total hip replacement. N Engl J Med. 2001;344:619-25.

19. Hansson PO, Sor'bo J, Eriksson H. Recurrent venous thromboembolism after deep vein thrombosis: incidence and risk factors. Arch Intern Med. 2000;160:769-74. https://doi.org/10.1001/archi nte.160.6.769.
20. Prandoni P, Noventa F, Ghirarduzzi A, et al. The risk of recurrent venous thromboembolism after discontinuing anticoagulation in patients with acute proximal deep vein thrombosis or pulmonary embolism: a prospective cohort study in 1,626 patients. Haematologica. 2007;92:199-205. https://doi.org/10.3324/haematol. 10516.

21. Kearon C, Akl EA, Ornelas J, et al. Antithrombotic therapy for VTE disease: CHEST guideline and expert panel report. Chest. 2016;149:315-52. https://doi.org/10.1016/j.chest.2015.11.026.

22. Boutitie F, Pinede L, Schulman S, et al. Influence of preceding length of anticoagulant treatment and initial presentation of venous thromboembolism on risk of recurrence after stopping treatment: analysis of individual participants' data from seven trials. BMJ. 2011;342: d3036. https://doi.org/10.1136/bmj.d3036.

23. Konstantinides SV, Torbicki A, Agnelli G, Task Force for the Diagnosis and Management of Acute Pulmonary Embolism of the European Society of Cardiology (ESC), et al. 2014 ESC guidelines on the diagnosis and management of acute pulmonary embolism. Eur Heart J. 2014;35(43):3033-69 (3069a-k).

24. Kyrle PA, Kammer M, Eischer L, et al. The long-term recurrence risk of patients with unprovoked venous thromboembolism: an observational cohort study. J Thromb Haemost. 2016;14:2402-9. https://doi.org/10.1111/jth.13524.

25. Van Rein N, Lijfering WM, Bos MH, et al. Objectives and design of BLEEDS: a cohort study to identify new risk factors and predictors for major bleeding during treatment with vitamin $\mathrm{K}$ antagonists. PLoS ONE. 2016;11(12): e0164485. https://doi.org/ 10.1371/journal.pone.0164485.

26. Migita K, Bito S, Nakamura M, et al. Venous thromboembolism after total joint arthroplasty: results from a Japanese multicenter cohort study. Arthritis Res Ther. 2014;16:R154.

27. Eriksson BI, Bauer KA, Lassen MR, Turpie AG. Fondaparinux compared with enoxaparin for the prevention of venous thromboembolism after hip-fracture surgery. N Engl J Med. 2001;345:1298-304.

28. Donath L, Lützner J, Werth S, et al. Efficacy and safety of venous thromboembolism prophylaxis with fondaparinux or low molecular weight heparin in a large cohort of consecutive patients undergoing major orthopaedic surgery-findings from the ORTHO-TEP registry. Br J Clin Pharmacol. 2012;74:947-58.

29. Eichinger S, Hron G, Bialonczyk C, et al. Overweight, obesity, and the risk of recurrent venous thromboembolism. Arch Intern Med. 2008;168(15):1678-83. https://doi.org/10.1001/archinte. 168.15.1678.

\section{Authors and Affiliations}

\section{Pinjala Ramakrishna ${ }^{1}$ P. C. Gupta ${ }^{2} \cdot$ Paresh Pai $^{3} \cdot$ Kumud Rai $^{4} \cdot$ M. Rajkumar ${ }^{5} \cdot$ Tapish Sahu $^{6}$. Mohammed Yunus Khan ${ }^{7} \cdot$ Kumar Gaurav $^{7}$. Amey Mane ${ }^{7}$. Sucheta Pandit ${ }^{7} \cdot$ Madhur Jain $^{8} \cdot$ Anand Subramaniyan $^{9}$}

\author{
Pinjala Ramakrishna \\ pinjala@hotmail.com \\ P. C. Gupta \\ pcgupta10@hotmail.com \\ Paresh Pai \\ drpareshpai@gmail.com \\ Kumud Rai \\ kumud.rai@maxhealthcare.com \\ M. Rajkumar \\ dr_rajkumar47@rediffmail.com
}

Tapish Sahu

tapish.sahu@gmail.com

Kumar Gaurav

Kumargaurav2@drreddys.com

Amey Mane

amey.mane@drreddys.com

Sucheta Pandit

Suchetaakshay.p@drreddys.com

Madhur Jain

dr.madhurjain1@gmail.com 
Anand Subramaniyan

anandsubbu81@yahoo.co.in

1 Apollo Hospitals, Jubilee Hills, Hyderabad, India

2 Vascular and Endovascular Surgery and Vascular Interventional Radiology, Care Hospital, Hyderabad, India

3 Bhatia Hospital, Mumbai, India

4 Max Healthcare, Delhi, India
5 Apollo Specialty Hospitals, Vanagaram, Chennai, India

6 Medanta Hospital, Gurugram, India

7 Dr.Reddy's Laboratories, Medical Affairs, Ameerpet, Hyderabad, Telangna, India

8 Ujala Cygnus Super Speciality Hospital, Rewari, Haryana, India

9 Kauvery Heartcity, Tamil Nadu, Tiruchirapalli, India 